\title{
The influence of problem features and individual differences on strategic performance in simple arithmetic
}

\author{
INEKE IMBO, ANDRÉ VANDIERENDONCK, AND YVES ROSSEEL \\ Ghent University, Ghent, Belgium
}

\begin{abstract}
The present study examined the influence of features differing across problems (problem size and operation) and across individuals (gender, amount of daily arithmetic practice, calculator use, and arithmetic skill) on simple arithmetic performance. Regression analyses were used to investigate the role of these variables in both strategy selection and strategy efficiency. Results show that more skilled and highly practiced students used memory retrieval more often and executed their strategies more efficiently than did less skilled and practiced students. Furthermore, calculator use correlated with both retrieval and procedural strategy efficiency but not with strategy selection. Only very small associations with gender were observed, with boys retrieving slightly faster than girls. Implications of the present findings for models of mental arithmetic are discussed.
\end{abstract}

Strategic performance of adults on cognitive problems consists of two main components. To solve a cognitive problem, people first have to choose the most appropriate strategy to solve it (i.e., strategy selection). Next, they have to execute the chosen strategy with reasonable speed and accuracy (i.e., strategy efficiency). For a long time, researchers studying mental arithmetic assumed that adults used only memory retrieval to solve simple arithmetic problems such as $8+3$ or $5 \times 4$ (see, e.g., Ashcraft, 1987, 1992, 1995; Campbell, 1987a, 1995; Campbell \& Oliphant, 1992; Lebiere \& Anderson, 1998; McCloskey, 1992; Siegler, 1989; Widaman \& Little, 1992). Fairly recently however, LeFevre and colleagues (LeFevre, Bisanz, et al., 1996; LeFevre, Sadesky, \& Bisanz, 1996; see also Baroody, 1994; Geary, Frensch, \& Wiley, 1993; Geary \& Wiley, 1991) showed that even skilled adults still make substantial use of procedures such as counting (e.g., $6+$ $3=6+1+1+1$ ) and transformation (e.g., $7+5=$ $7+3+2$ ) when solving simple arithmetic problems. It is clear that retrieval and nonretrieval (i.e., procedural) strategies differ in their efficiency, since retrieval is generally much faster (i.e., more efficient) than any procedural strategy. Although people may adopt other strategies to solve arithmetic problems (such as using a calculator), the present study investigated mental arithmetic and thus focused on the two broad kinds of strategy mentioned above - retrieval and procedural.

Both strategy selection and strategy efficiency may depend on factors such as problem features (e.g., operation, problem size) and individual differences ${ }^{1}$ (e.g., arithmetic skill, arithmetic practice). Although models have been proposed in which such experiential factors are the main determinants of mental representation, acquisition, and performance (see, e.g., Ashcraft, 1987; Campbell \& Graham, 1985; Siegler, 1988; Siegler \& Shipley, 1995), there are very few direct comparisons of the simple arithmetic performance of adults who differ in their mathematical education, arithmetic skill, or arithmetic practice (see also LeFevre \& Liu, 1997). Moreover, up until now, no study has investigated the effects of these factors in strategy selection and strategy efficiency separately. The present study therefore examined the effects of features that differed across problems and across individuals on strategic performance of simple arithmetic.

\section{Problem Features}

Although adults rely on both retrieval and procedural strategies for the entire domain of elementary arithmetic (i.e., the four basic operations; see, e.g., Campbell \& Xue, 2001), they adjust their strategy selection according to the operation involved. In solving subtraction and division problems, for example, adults rely more heavily on procedural strategies than they do when solving either addition or multiplication problems, for which retrieval strategies are predominantly used (Campbell \& Xue, 2001; Seyler, Kirk, \& Ashcraft, 2003). Furthermore, adults use direct retrieval to solve multiplication problems even more frequently than they do to solve addition problems (see, e.g., Hecht, 1999; LeFevre, Bisanz, et al., 1996; LeFevre, Sadesky, \& Bisanz, 1996). This might be explained by the fact that solving multiplication problems depends for the most part on declarative knowledge, whereas solving addition problems depends on both declarative and procedural knowledge (Roussel, Fayol, \& Barrouillet, 2002).

I. Imbo, ineke.imbo@ugent.be 
Whatever the operation is, strategy selection also depends on the size of the problem. For smaller problems (e.g., $2+4)$, people generally retrieve answers from their longterm memory, but for larger problems (e.g., $8+6$ ), they are more inclined to use procedural strategies.

Because procedural strategies are less efficient than retrieval strategies, longer latencies and higher error rates are observed on larger problems. This very robust effect is known as the problem size effect: As problem size increases, solution times and error percentages also increase. More frequent retrieval use on small than on large problems is not the only source for the problem size effect, however. According to Campbell and Xue (2001), as many as three sources account for this effect: more frequent use of procedural strategies for large than for small problems, less efficient retrieval strategies for large than for small problems, and less efficient procedural strategies for large than for small problems. Less efficient retrieval strategies can be explained by weaker associative connections between problem-answer pairs in the retrieval network (see, e.g., Siegler, 1988), whereas less efficient procedural strategies can be explained by the larger number of suboperations to be performed. Nevertheless, the problem size effect does not derive solely from strategic sources; the structure of the mental network (with different network strengths and spreading activation characteristics) and interference from competing associations are other contributing factors (see, e.g., Zbrodoff, 1995).

\section{Individual Differences}

Strategy selection and strategy efficiency do not depend only on problem features, but also on individual traits and culture-based factors. Several studies found differences between East Asians' and North Americans' performance on simple arithmetic (see, e.g., Campbell \& Xue, 2001; Chen \& Uttal, 1988; Geary, 1996; Geary, BowThomas, Liu, \& Siegler, 1996; Geary et al., 1997; Geary, Liu, \& Bow-Thomas, 1992; Geary, Salthouse, Chen, \& Liu, 1996; LeFevre \& Liu, 1997; Penner-Wilger, LethSteensen, \& LeFevre, 2002; Stevenson, Chen, \& Lee, 1993; Stevenson et al., 1990; Stevenson, Lee, \& Stigler, 1986). Because East Asians rely primarily on memory retrieval, their arithmetic performance is faster and less error prone than that of North Americans, who frequently use procedures. Arithmetic performance differences are found not only across cultures but also within one culture. People may differ from each other in several respects, such as level of mathematical education, daily arithmetic practice, arithmetic skill, and so forth. However, differences in adults' cognitive processes in arithmetic have not been extensively researched. Below, we summarize the main results of studies examining effects of cognitive factors on arithmetic performance. We also consider the role of gender.

Differences in arithmetic performance have been found to depend on arithmetic skill. LeFevre and Bisanz (1986), for example, found that low-skill people used less efficient and slower mental calculation processes than highskill people. Therefore, the difference between low- and high-skill subjects was greater on items that required cal- culations than on items that could be solved without calculations. Furthermore, LeFevre and colleagues (LeFevre, Bisanz, et al., 1996; LeFevre, Sadesky, \& Bisanz, 1996) observed more frequent retrieval use in high-skill than in low-skill people. More recently, high-skill subjects were shown to be more efficient (i.e., faster) in solving simple arithmetic problems than were low-skill subjects (Campbell \& Xue, 2001; Kirk \& Ashcraft, 2001; see also Geary \& Widaman, 1987; Gilles, Masse, \& Lemaire, 2001). Finally, LeFevre and colleagues (LeFevre \& Kulak, 1994; LeFevre, Kulak, \& Bisanz, 1991) found evidence for individual differences in the obligatory activation of addition facts; since associative connections are stronger in highskill subjects than in low-skill subjects, they concluded that accessibility of arithmetic facts may contribute to individual differences in solving arithmetic problems.

Besides arithmetic skill, strategic performance may also depend on other factors such as math attainment, daily arithmetic practice, and calculator use. Hecht (1999), for example, showed that adults with higher levels of math attainment used retrieval strategies more frequently, were more accurate in solving math facts, and retrieved arithmetic problems faster than adults with lower levels of math achievement. Roussel et al. (2002) found that people with high levels of daily arithmetic practice (primary school teachers) exhibited smaller problem size effects than people with low levels of daily arithmetic practice (undergraduate psychology students). However, the highly practiced subjects did not differ from the less practiced subjects in the strategies that they used (i.e., strategy selection). The frequency of calculator use might be another influencing factor. From primary school on, children are taught how to use a hand-held calculator, even though calculators are at the center of several controversies, both educational (i.e., is it good for children to use calculators?) and conceptual (i.e., are calculators designed and implemented well? See Thimbleby, 2000). Yet very few studies have investigated effects of calculator use on simple arithmetic performance. Campbell and Xue (2001) observed no reliable effect of the frequency of calculator use on simple arithmetic strategy selection or strategy efficiency.

Gender differences have been found in young children's arithmetic strategy selection (see, e.g., Carr \& Jessup, 1997; Carr, Jessup, \& Fuller, 1999; Fennema, Carpenter, Jacobs, Franke, \& Levi, 1998). Specifically, girls are more likely to use procedural strategies, whereas boys are more likely to use retrieval strategies. These gender differences in simple arithmetic strategy selection have been shown to be driven not only by skill differences but also by girls' and boys' strategy preferences (Carr \& Davis, 2001). Furthermore, gender differences have been found in retrieval efficiency as well, with boys being faster than girls from fifth grade on (Royer, Tronsky, Chan, Jackson, \& Marchant, 1999). Carr and Davis, however, observed no differences between boys and girls in retrieval efficiency. Although no study has explicitly investigated whether gender differences in strategy selection and strategy efficiency exist in adults, Geary, Saults, Liu, and Hoard (2000) reanalyzed simple arithmetic performance data obtained in a study by Geary et al. (1993) and observed more frequent retrieval 
use by men than by women ( $86 \%$ vs. $66 \% ; p<.07)$ but no gender differences in retrieval efficiency.

\section{Overview of the Present Study}

As described earlier, problem features have large effects on adults' simple arithmetic performance. Individual differences, however, have been studied less frequently. For example, gender effects in adults' simple arithmetic performance have not directly been investigated thus far. Effects of the frequency of calculator use have not been observed either. The effects of arithmetic practice over and above those of arithmetic skill are still debated as well. Moreover, even when individual differences in simple arithmetic performance have been studied, no distinction between their roles in strategy selection and strategy efficiency has been made. Therefore, the present study investigated effects of both problem features and individual differences on simple arithmetic strategy selection and strategy efficiency separately. In addition to the investigation of two typical problem features (problem size and operation), effects of four individual traits were tested: daily arithmetic practice, arithmetic skill, amount of calculator use, and gender. The novelty here is the distinction between skill and practice. Whereas most previous studies selected low- and high-skill subjects on the basis of arithmetic subtests only (see, e.g., Gilles et al., 2001; Hecht, 1999; LeFevre \& Bisanz, 1986; LeFevre, Sadesky, \& Bisanz, 1996), the present study incorporated the amount of subjects' daily arithmetic practice in addition to the measure of their arithmetic skill. In fact, the amount of daily arithmetic practice was assessed on the basis of the students' high school curricula, which all differed in the number of required math and science classes per week. The Belgian education system, in which high school students must choose one main subject every 2 years, offers a good opportunity to investigate such practice effects in an ecologically valid way. Indeed, all students in the present study were enrolled in a specific curriculum with a fixed number of math classes each week. In contrast, arithmetic skill was measured by means of a frequently used penand-paper test (the French Kit). A short questionnaire determined each student's habits concerning calculator use.

On the basis of previous research, we expected more frequent retrieval use and more efficient strategy use in the high-skill than in the low-skill students. We also expected simple arithmetic performance to be related to daily arithmetic practice, with more frequent retrieval use and more efficient strategy execution in the more practiced students than in the less practiced students. Although there is no evidence for effects of calculator use on simple arithmetic performance (Campbell \& Xue, 2001), we expected less frequent retrieval use and less efficient strategy use for students who used the calculator frequently than for students who rarely used it. It was difficult to make any predictions regarding gender differences, since such differences are more pronounced in children than in adults. However, on the basis of developmental studies on boys' and girls' arithmetic performance, if any differences were to appear in the present study, we expected more frequent and more efficient retrieval use in male than in female students.

\section{METHOD}

\section{Subjects}

Sixty sixth-year ${ }^{2}$ high school students participated in this study. The amount of these students' daily arithmetic practice was assessed on the basis of the number of hours per week they spent in math and science classes. Science classes were considered because they frequently require the use of arithmetic. The number of hours per week students spent in math and science classes was 3 (14 females and 1 male; mean age 17 years, 8 months); 4 (14 females and 2 males; mean age 17 years, 7 months); 9 ( 1 female and 1 male; mean age 17 years, 6 months), 11 (4 females and 4 males; mean age 17 years, 9 months), 13 ( 1 female and 9 males; mean age 17 years, 6 months), and 15 ( 5 females and 4 males; mean age 17 years, 8 months). At the time of measurement, all students had been enrolled in their specific curriculum for at least 1.5 years. All students participated voluntarily, with the permission of their parents and the school teachers.

\section{Procedure and Stimuli}

Each subject was tested individually in a quiet classroom for approximately $45 \mathrm{~min}$. The test session started with short questions about the subject's age, curriculum, and the number of math and science classes taken per week. Three tasks were given to each subject. The first was the simple arithmetic task, which consisted of two blocks, one with addition problems and one with multiplication problems, the order of which was counterbalanced across subjects. Subsequently, an arithmetic skill test (the French Kit) was administered. The session ended with a short questionnaire about calculator use.

Simple arithmetic task. The stimuli for this task consisted of simple addition and multiplication problems. Following previous studies, we used the so-called standard set of problems (LeFevre, Sadesky, \& Bisanz, 1996), which excludes problems involving 0 or 1 as an operand or answer. Both addition and multiplication problems were composed of pairs of numbers between 2 and 9 , with tie problems (e.g., $3+3$ ) excluded; commuted pairs (e.g., $2+4$ and $4+2$ ) were considered to be two different problems, resulting in 56 addition problems (ranging from $2+3$ to $8+9$ ) and 56 multiplication problems (ranging from $2 \times 3$ to $8 \times 9$ ). Problem size was defined according to the correct answer to the problem (from 5 to 17 for the sums and from 6 to 72 for the products). This continuous definition of problem size differs from the widely used dichotomous definition of problem size (i.e., a categorization into small and large problems).

A trial started with a fixation point, which appeared on the computer screen for $500 \mathrm{msec}$. Then the arithmetic problem appeared in the center of the screen. The addition and multiplication problems were presented horizontally in Arabic format as dark blue characters on a light gray background, with the operation sign $(+$ or $\times)$ at the fixation point. The problem remained on the screen until the subject responded. Although subjects were required to respond as quickly and as accurately as possible, no time deadline was set because it has been shown that a tight deadline increases reported use of retrieval, especially for large problems (Campbell \& Austin, 2002). Timing began when the stimulus appeared and ended when the response triggered the sound-activated relay. To enable this sound-activated relay, subjects wore a microphone, which was activated when they spoke their answer aloud. This microphone was connected to a software clock accurate to $1 \mathrm{msec}$. On each trial, subjects received feedback in the form of the word "correct" appearing in green on the screen, or the word "fout" (Dutch for "wrong") appearing in red.

Subjects were also told to report the strategy that they used for each problem. They could choose one of the following strategy categories: retrieval, counting, transformation, or other (see, e.g., Campbell \& Gunter, 2002; Campbell \& Xue, 2001; Kirk \& Ashcraft, 2001; LeFe- 
vre, Sadesky, \& Bisanz, 1996; Seyler et al., 2003). At the beginning of the experiment, each strategy was described as follows.

(1) Retrieval: You solve the problem by just remembering or knowing the answer directly from memory. It means that you know the answer without any additional processing, or that the answer "pops into your head." (2) Counting: You solve the problem by counting a certain number of times to get the answer (e.g., $6+3=6 \ldots 7 \ldots 8 \ldots 9 ; 3 \times 6=6 \ldots 12 \ldots 18$ ). (3) Transformation:You solve the problem by referring to related operations or by deriving the answer from some known facts. You change the presented problem to take advantage of a known arithmetical fact (e.g., $6+7=6+6+1 ; 9 \times 6=60-6$ ). (4) Other:You solve the problem by using a strategy unlisted here (e.g., guessing), you used more than one strategy, or you do not know what strategy you used to solve the problem.

After each trial, the four category names were displayed on the screen. The subject also kept a copy of the strategy report instructions for reference during the study. It was emphasized that the presented strategies were not meant to encourage use of a particular strategy.

The subject's answer, the reported strategy, and the validity of the trial were recorded online by the experimenter. All invalid trials (such as those on which the voice-activated relay failed) were discarded and rerun at the end of the block. This procedure enabled us to minimize data loss due to unwanted failures. Each block (addition or multiplication problems) started with four practice problems, followed by the experimental problems. Since each problem was presented twice, each block consisted of 112 arithmetic trials, which were presented in a random order. After the first block and a short break, the second block (with the other operation) was administered, consisting of four practice problems and 112 experimental problems as well.

French Kit. After the simple arithmetic task, subjects completed two arithmetic subtests of the French Kit (Ekstrom, French, \& Harman, 1976; French, Ekstrom, \& Price, 1963), consisting of one page of complex addition problems and one page of complex subtraction and multiplication problems. Each page contained six rows of 10 vertically oriented problems. Each subject was given 2 min per page to solve the problems as quickly and accurately as possible. Arithmetic skill was defined as the total number of problems solved correctly on both tests. This measure of arithmetic skill reflects the ability to execute strategies on multidigit problems quickly and accurately. The French Kit is also used to measure arithmetic fluency and working memory management (Geary \& Widaman, 1992).

Calculator use questionnaire. Subjects received a page on which the following question was written: "How often did you use a calculator (or another electronic device such as a cell phone) when doing arithmetic problems (e.g., $65+34,23 \times 17)$ ?" Subjects had to provide an answer to this question by marking a 5-point rating scale ranging from never to always, once concerning their experiences during elementary school and once concerning their experiences during high school.

\section{RESULTS}

Overall, 1,305 trials (i.e., 9.09\%) were spoiled due to failures of the sound-activated relay. Since all of these invalid trials returned at the end of the block, most of them were recovered from data loss, which reduced the trials spoiled due to failures of the sound-activated relay to 425 (i.e., 2.96\%). Further, all incorrect trials and all trials on which subjects selected "other" as their strategy were deleted (a total of $3.03 \%$ ). Finally, all response times (RTs) more than four standard deviations from each individual subject's mean (per operation) were discarded as outliers $(0.5 \%$ for addition and $1.1 \%$ for multiplication). The final data set consisted of 13,026 valid trials, which corresponds to a total data loss of less than $8 \%$. In the following, all reported results are considered to be significant if $p<.05$, unless mentioned otherwise.

Regression analyses were performed to detect which factors contributed to strategy selection and strategy efficiency. Separate regression analyses were run for addition and multiplication problems on the three dependent variables: percentage retrieval use, retrieval RTs, and procedural RTs (see Table 1). The predictors in all regression analyses were (1) problem size (defined according to the correct answer to the arithmetic problem); (2) daily arithmetic practice (defined as the number of math and science classes per week); (3) calculator use (as measured by the questionnaire); (4) arithmetic skill (as measured by the French Kit test); and (5) gender (male or female). The first predictor varied across problems, whereas the other variables varied across individuals. Table 2 describes means, medians, minima, and maxima of the independent variables varying across individuals (except gender). ${ }^{3}$ A paired-samples $t$ test showed that calculator use was more frequent in high school than in elementary school $[t(59)=13.40, S E=.157$, with mean scores of $4.0(S D=0.88)$ and $1.9(S D=0.90)$, respectively]. Since we expected that only the current frequency of calculator use would influence strategic performance, the frequency of calculator use in high school was included in the regression analyses, whereas the frequency of calculator use in elementary school was not. A summary of all the regression analyses is presented in Table 3.

The $R^{2} \mathrm{~s}$ for percentage retrieval use were .298 $\left[F(5,774)=65.72, M S_{\mathrm{e}}=764.08\right]$ for addition problems and $.164\left[F(5,1493)=58.67, M S_{\mathrm{e}}=639.55\right]$ for multiplication problems. For both operations, retrieval use oc-

Table 1

Means, Standard Deviations (SDs), Medians, Minima, and Maxima of the Three Dependent Variables Used in the Regression Analyses

\begin{tabular}{lrrrrrrrr}
\hline & \multicolumn{2}{c}{ Retrieval Use (\%) } & & \multicolumn{2}{c}{ Retrieval RTs (msec) } & & Procedural RTs (msec) \\
\cline { 2 - 3 } & Add. & Mult. & & Add. & Mult. & & Add. & Mult. \\
\hline Mean & 78 & 87 & & 874 & 1,376 & & 1,114 & 2,865 \\
SD & 4 & 3 & & 296 & 688 & & 680 & 2,368 \\
Median & 81 & 89 & & 857 & 1,343 & & 1,035 & 2,184 \\
Minimum & 31 & 53 & & 601 & 689 & & 710 & 889 \\
Maximum & 100 & 100 & & 1,382 & 2,573 & & 2,464 & 7,453 \\
\hline
\end{tabular}

Note-Add., addition; Mult., multiplication. 
Table 2

Means, Medians, Minima, and Maxima of the Individual Characteristic Variables (Except Gender) Used As Predictors in the Regression Analyses

\begin{tabular}{lcccc}
\hline & Mean & Median & Minimum & Maximum \\
\hline Daily arithmetic practice (hours) & 8 & 4 & 3 & 15 \\
Calculator use in high school* $^{*}$ & 4 & 4 & 1 & 5 \\
Calculator use in elementary school* & 2 & 2 & 1 & 5 \\
Arithmetic skill (score on the French Kit) & 28 & 26 & 15 & 58 \\
*The frequency of calculator use in both elementary school and high school was deter- \\
mined; however, only the current frequency of calculator use (i.e., in high school) was used \\
in the regression analyses.
\end{tabular}

curred more frequently with smaller problem sizes and higher arithmetic skill. More frequent daily practice predicted more frequent retrieval use only for multiplication problems. The regression analyses on retrieval RTs resulted in an $R^{2}$ of $.322\left[F(5,722)=68.51, M S_{\mathrm{e}}=48,515.37\right]$ for addition problems and an $R^{2}$ of $.248[F(5,1417)=93.51$, $\left.M S_{\mathrm{e}}=307,053.80\right]$ for multiplication problems. For both operations, answers were retrieved faster with smaller problem size, higher arithmetic skill, and less frequent calculator use. More extensive daily practice predicted faster retrieval use only for multiplication problems. Furthermore, boys tended to be slightly faster in retrieving multiplication facts than girls. Finally, for procedural RTs, an $R^{2}$ of .175 $\left[F(5,328)=13.87, M S_{\mathrm{e}}=240,580.01\right]$ was obtained for addition problems, and an $R^{2}$ of $.229[F(5,350)=20.77$, $\left.M S_{\mathrm{e}}=4,013,325.39\right]$ for multiplication problems. For both operations, procedural strategies were performed faster when problem size was smaller, arithmetic skill was higher, and calculator use was less frequent. Again, more daily practice predicted faster procedural use for multiplication problems but not for addition problems.

\section{DISCUSSION}

Results of the present study show that Belgian high school students used a variety of strategies to solve simple arithmetic problems, which is in accordance with comparable research in non-European subjects (see, e.g., Campbell \& Xue, 2001; Hecht, 1999; LeFevre \& Liu, 1997; LeFevre, Bisanz, et al.,1996; LeFevre, Sadesky, \& Bisanz, 1996), and, as LeFevre and collaborators reported, sharply in contrast with the assumption that adults always retrieve arithmetic facts from memory. There are, however, both similarities and differences between the present results and previous findings. We observed $78 \%$ retrieval use for addition and $87 \%$ retrieval use for multiplication. These percentages are at the high end of the range of percentages observed in North Americans, which ranged from $66 \%$ to $76 \%$ for addition and from $59 \%$ to $96 \%$ for multiplication (see, e.g., Campbell \& Xue, 2001; Campbell \& Timm, 2000; Geary, 1996; Hecht, 1999; LeFevre, Bisanz, et al., 1996; LeFevre, Sadesky, \& Bisanz, 1996), but they are well below the percentages observed in East Asians, whose percentage of retrieval use for addition was $92 \%$ and for multiplication, $100 \%$ (see, e.g., Campbell \& Xue, 2001; Geary, 1996; LeFevre \& Liu, 1997). The number of subjects using retrieval on all trials was $6(10 \%)$ for addition and $16(26 \%)$ for multiplication; these figures are comparable to those of LeFevre, Bisanz, et al. (1996) and LeFevre, Sadesky, and Bisanz (1996), in which $12.5 \%$ and $28 \%$ of the subjects used retrieval on all trials for addition and multiplication, respectively.

Table 3

Summary of the Regression Analyses for Variables Predicting Percentage Retrieval Use, Retrieval RTs, and Procedural RTs

\begin{tabular}{|c|c|c|c|c|c|c|}
\hline & \multicolumn{3}{|c|}{ Addition } & \multicolumn{3}{|c|}{ Multiplication } \\
\hline & $B$ & $S E$ & $\beta$ & $B$ & $S E$ & $\beta$ \\
\hline \multicolumn{7}{|l|}{ Retrieval Use } \\
\hline Problem size & -4.348 & 0.265 & $-.495^{* *}$ & -0.595 & 0.037 & $-.380^{* * *}$ \\
\hline Arithmetic skill & 0.697 & 0.131 & $.174^{* *}$ & 0.367 & 0.087 & $.109^{* *}$ \\
\hline Calculator use & -0.099 & 0.052 & -.066 & 0.065 & 0.034 & .052 \\
\hline Daily practice & 0.044 & 0.244 & .006 & 0.422 & 0.161 & $.074^{* *}$ \\
\hline Gender & 3.061 & 2.614 & .044 & 0.732 & 1.725 & .013 \\
\hline \multicolumn{7}{|l|}{ Retrieval RTs } \\
\hline Problem size & 30.873 & 2.231 & $.424^{* *}$ & 14.177 & 0.849 & $.385^{* *}$ \\
\hline Arithmetic skill & -9.206 & 1.068 & $-.288^{* *}$ & -18.302 & 1.930 & $-.238^{* *}$ \\
\hline Calculator use & 1.510 & 0.423 & $.125^{* *}$ & 2.037 & 0.779 & $.069^{* *}$ \\
\hline Daily practice & -1.011 & 2.042 & -.018 & -7.751 & 3.625 & $-.059^{*}$ \\
\hline Gender & -38.779 & 22.061 & -.070 & -80.402 & 38.564 & $-.060^{*}$ \\
\hline \multicolumn{7}{|l|}{ Procedural RTs } \\
\hline Problem size & 40.437 & 9.270 & $.220^{* *}$ & 19.154 & 6.203 & $.146^{* *}$ \\
\hline Arithmetic skill & -9.072 & 3.740 & $-.138^{* *}$ & -108.738 & 17.571 & $-.309^{* *}$ \\
\hline Calculator use & 3.401 & 1.740 & $.127^{*}$ & 12.721 & 5.235 & $.146^{* *}$ \\
\hline Daily practice & -13.553 & 7.366 & -.114 & -103.214 & 28.510 & $-.214^{* *}$ \\
\hline Gender & -131.687 & 76.057 & -.109 & 344.256 & 346.783 & .070 \\
\hline
\end{tabular}

${ }^{*} p<.05 . \quad{ }^{* *} p<.01$. 
Beyond confirming previous results, the present study yielded several new findings concerning individual differences in strategy selection and strategy efficiency. Concerning strategy selection, direct memory retrieval was used more often by high-skill students than by low-skill students; it was also used more often by more practiced students than by less practiced students, although the latter was true only for multiplication problems, not for addition problems. Note that some previous researchers did observe individual differences in strategy selection (e.g., Hecht, 1999; LeFevre, Sadesky, \& Bisanz, 1996), whereas others did not (e.g., Roussel et al., 2002). Strategy efficiency also differed across individuals. Both retrieval efficiency and procedural efficiency increased with the level of arithmetic skill and the level of daily arithmetic practice, although, again, the latter was true for multiplication but not for addition. Remarkably, the frequency of calculator use influenced strategy efficiency as well: Students who reported frequent calculator use showed lower retrieval and procedural efficiency levels but did not differ in strategy selection. Gender correlated only with retrieval efficiency, with the finding that boys were slightly faster than girls at retrieving multiplication facts from memory. Below, we first elaborate on the observed individual differences and then describe some implications for the present models of mental arithmetic.

\section{Daily Arithmetic Practice}

Since most previous studies examined individual differences by means of pen-and-paper tests, the present study also incorporated a more ecological variable - daily arithmetic practice. The amount of the subjects' daily arithmetic practice was calculated on the basis of the number of their math and science classes per week during the past years in high school. As outlined in the introduction, practice effects were expected on strategy selection, retrieval efficiency, and procedural efficiency. All of these hypotheses were confirmed: All three measures of simple arithmetic strategic performance were significantly linked to arithmetic practice, albeit only for multiplication problems.

The finding that less practiced students used retrieval less often than more practiced students provides support for previous studies that assumed that practice leads to increases in retrieval frequency (see, e.g., Siegler, 1988; Widaman \& Little, 1992). Although arithmetic practice influenced strategy selection, retrieval and procedural strategies were used regardless of the level of practice. Moreover, the more practiced students used retrieval more efficiently than the less practiced students. For problems that were practiced frequently, students may have developed stronger connections between operands and correct answers than they had for less frequently practiced problems (see, e.g., Siegler, 1988), and these stronger problem-answer associations may have resulted in faster retrieval use. Previous studies indeed showed that associative connections may differ across individuals (LeFevre \& Kulak, 1994; LeFevre et al., 1991). Procedural efficiency was much higher for the more practiced students than for the less practiced students. LeFevre, Bisanz, et al. (1996) stated that practice may lead not only to more frequent re- trieval use, but also to automatic activation of procedural strategies. If the successful use of procedural strategies increases the strength of the problem-procedure association, practice may thus also influence procedural efficiency, an effect that was observed here. More practiced students may thus have both stronger problem-answer associations and problem-procedure associations, resulting in higher retrieval and procedural efficiencies, respectively. Because practice enhances procedural efficiency, procedural strategies can be maintained as alternatives of equal value to retrieval strategies. Distributions of problem-answer and problem-procedure associations should thus be viewed as continuously dynamic, rather than as reaching a final static state (LeFevre, Bisanz, et al., 1996).

But why were the effects of arithmetic practice significant for multiplication problems but not for addition problems? One explanation for this operation-dependent effect is that strategy efficiencies differ between addition and multiplication. More specifically, compared with retrieval strategies, procedural strategies are less efficient for multiplication than they are for addition (Campbell \& Xue, 2001). Because people always try to select the most efficient strategy (Siegler \& Shipley, 1995), they will especially limit the use of procedures in order to solve multiplication problems. In other words, they will try to use retrieval more often, and more so for multiplication than for addition. Daily arithmetic practice may enhance this effect. Indeed, more practiced students did retrieve multiplication problems more frequently than did less practiced students. Moreover, since procedures are less efficient for multiplication than for addition, multiplication procedures are more susceptible to amelioration than addition procedures are. Two other factors may account for the operation-dependent effect on strategy efficiency as well: the more frequent use of multiplication in math and science classes and the more declarative nature of multiplication (Roussel et al., 2002). Consequently, the more practiced students may have built up stronger problem-answer and problem-procedure associations for multiplication than for addition, resulting in higher retrieval efficiency and higher procedural efficiency, respectively.

\section{Arithmetic Skill}

Arithmetic skill was measured by means of a pen-andpaper test and thus differed from the practice measure, which was based on number of math and science classes per week. According to LeFevre, Bisanz, et al. (1996), arithmetic skill can be viewed on a continuum from novice to expert, with high-skill subjects retrieving arithmetic facts more frequently and more efficiently than lowskill subjects (see also Ashcraft, Donley, Halas, \& Vakali, 1992; Kaye, 1986; Kaye, deWinstanley, Chen, \& Bonnefil, 1989; LeFevre \& Kulak, 1994); these effects were observed in the present study as well. Indeed, regression analyses showed that arithmetic skill was the only individual trait that was highly predictive both of use of a retrieval strategy and of strategy efficiency in both addition and multiplication problems. One may thus conclude that both strategy selection and strategy efficiency are potentially important indexes of individual differences in skill. 
Differences across our subjects may also be compared with differences across cultures. LeFevre and Liu (1997) showed that East Asian students differed from North American students in retrieval efficiency, procedural efficiency, and retrieval use as well. LeFevre and Liu did not conclude, however, that these differences between East Asians and North Americans were due to overall differences in arithmetic skill. They state that if arithmetic skill differences were the cause, "a comparison between any groups of less- and more-skilled individuals would yield similar patterns of effects" (p. 51). LeFevre and Liu therefore supposed that fundamental differences in the organization of basic arithmetic facts in memory were responsible for the observed differences between East Asian and North American students. In the present study, we did observe patterns across our same-culture subjects comparable to patterns that have been observed across cultures (i.e., differences in both strategy selection and strategy efficiency). This result might imply that our same-culture subjects differed in their organization of basic arithmetic facts in memory, an issue that merits future research.

Finally, it should be noted that the influence of arithmetic skill was greater than the influence of daily arithmetic practice. One may thus argue that arithmetic skill largely determines simple arithmetic performance, with another, more experience-based individual trait, such as daily practice, perhaps playing a smaller role. To what extent arithmetic skill may depend on variable factors such as daily arithmetic practice and on more stable factors such as general intelligence is an issue that future researchers may pursue. Future researchers may also pursue disentangling the effects of arithmetic skill from those of arithmetic practice, possibly by controlling arithmetic skill and manipulating the amount of practice.

\section{Frequency of Calculator Use}

Although cultural differences in the frequency of calculator use have been found (LeFevre \& Liu, 1997), this study was the first to relate differences in calculator use to arithmetic performance within one single culture. A first observation was that the frequency of calculator use increases as children grow older (see also Campbell \& Xue, 2001). One may further assume that this frequency will increase even more, since nowadays most people (even children) always have cell phones, which often include calculators, at hand. This frequent use of calculators may pose problems, however. The present study showed a negative relationship between calculator use and strategy efficiency: The efficiency of both retrieval and procedural strategies diminished as the frequency of calculator use increased. This assertion should be treated with caution, however, since regression analyses simply showed a relationship between calculator use on the one hand and strategy selection and strategy efficiency on the other; they did not reveal the direction of causality. Future studies are thus needed to investigate whether frequent calculator use results in poorer arithmetic performance or whether students poorer in mental arithmetic are more inclined to use a calculator.

\section{Gender Differences}

Although we did not really expect to observe differences between our young male and female adults in simple arithmetic performance, regression data indicated that males were somewhat more efficient at retrieving multiplication facts from memory than females were. Since any cognitive difference between males and females is of scientific and social importance, this issue merits further discussion. Royer and his colleagues (1999) also observed more efficient retrieval use in boys than in girls, but the present study is the first one to observe comparable effects in young adults. Several researchers, including Geary (1999) and Royer et al. (1999) have argued that any gender difference in retrieval efficiency is not likely to result directly from biological factors such as sex hormones or primary memory systems. Instead, Geary (1999) proposed that gender might indirectly influence differences in mathematical cognition. Specifically, he proposed that the cognitive systems enabling movement in and the representation of 3-D space are more highly elaborated in males than in females. Since these brain systems may support mathematical cognition, they may account for the observed differences in mental arithmetic between males and females. Further testing confirmed that the male advantage in mental arithmetic was mediated by gender differences, in both spatial abilities and retrieval efficiency, that favored males (Geary et al., 2000).

It should be noted, however, that arithmetic performance is not determined only by retrieval efficiency, but also by procedural efficiency and retrieval frequency; results from the present study indicate that males and females would not differ in the latter respects. Further research, however, is needed to confirm or reject gender as a factor in retrieval speed and to further investigate sources of these and other differences between males and females in various cognitive domains.

\section{Other Variables}

Although the present study examined various individual differences, others still remain unexamined. We chose to focus on cognitive differences across people, who may also vary in emotionality. Previous studies have indeed shown that strategic aspects of simple arithmetic might be influenced by people's attitude toward mathematics (LeFevre, Bisanz, et al., 1996) and by their math anxiety (Ashcraft \& Faust, 1994; Ashcraft \& Kirk, 2001; Faust, Ashcraft, \& Fleck, 1996). If we assume that high-anxiety students choose curricula with fewer hours of mathematics whereas low-anxiety students choose curricula with more hours of mathematics, such emotional factors should be seen as confounding variables, because they were not controlled in the present study. In addition to such effects of self-selection, the effects of parental selection should not be denied either. It can be assumed that parents influence their children to choose a study curriculum heavy or light on math and science courses. It would be worthwhile to investigate whether arithmetic practice interacts with the enthusiasm with which students have chosen their study curriculum. Finally, general intelligence might have been an additional confounding variable. As with the factors 
mentioned earlier, it is difficult to control for intelligence. The present study thus cannot exclude its effects completely. Future research should aim at disentangling effects of emotional, parental, and cognitive factors (including general cognitive ability) on strategic performance in simple arithmetic.

\section{Implications for Simple Arithmetic Models}

When solving simple arithmetic problems, use of multiple strategies appears to be common in young adults (see also Campbell \& Xue, 2001; Hecht, 1999; LeFevre \& Liu, 1997; LeFevre, Bisanz, et al., 1996, LeFevre, Sadesky, \& Bisanz, 1996). Most present models of mathematical cognition simply account for retrieval-only data, and therefore such models cannot account for all simple arithmetic performance in adults; a multiple-procedure model is required. The majority of current models cannot explain multiple-strategy use, or have not included experiential or educational factors in order to explain performance differences across subjects, or both. Measures of associative strength, for example, may be associated with individual differences such as daily arithmetic practice or arithmetic skill. However, as Hecht (1999) noted, most associative network models assume that the problem-answer associations are quite similar among adults because adults all share common experiences that influence the formation of their associative networks. These "common experiences" refer to how math facts are practiced and studied in childhood. The present study, however, showed that effects of experience do not stop after childhood, since practice still influenced simple arithmetic performance in the young adults who participated in our study. Incorporating the ongoing development of human beings would truly enrich models of mental arithmetic. Together with other researchers (e.g., LeFevre, Bisanz, et al., 1996; LeFevre \& Liu, 1997), we thus believe that theories of mathematical cognition should include the possibility that reasonably skilled adults with different experiential backgrounds may vary in patterns of performance. Therefore, adequate models of mental arithmetic must make additional assumptions to account for both retrieval and nonretrieval responses and must incorporate a role for individual differences and their consequences on arithmetic performance.

One model that, with some small modifications, could account for our data and those of other researchers (e.g., LeFevre, Bisanz, et al., 1996) is the adaptive strategy choice model (ASCM; Siegler \& Shipley, 1995). According to this model, problems, strategies, and problemstrategy strengths are stored in a database. Each time a problem is solved, information about the efficiency of the strategy (e.g., its time and accuracy) is added to the database, which is thus modified. The probability of choosing a particular strategy is based on the strategy's strength relative to the strength of all other strategies. Furthermore, each time a particular strategy is used to solve a problem, the association between the problem and the strategy used is strengthened. In such a model, adults are no longer assumed to rely solely on retrieval. If we further assume that the amount of daily arithmetic practice influences problem-strategy associations (e.g., practice might strengthen the problem-retrieval association and weaken the problem-procedure association), ASCM would predict performance differences across subjects with different amounts of practice. Therefore, we do not propose that the basic structure of the models should be changed, but that they should be updated by not only including problem features (e.g., problem size), but also strategy selection characteristics (e.g., single- or multiple-strategy use), and individual traits (e.g., arithmetic skill, arithmetic practice) in order to develop complete models of mental arithmetic that are able to explain various effects in simple arithmetic performance. Previous studies have already suggested some ideas for modifications to present models (see, e.g., Hecht, 1999; LeFevre et al., 1996).

\section{CONCLUSIONS}

Although it has been suggested that experiential factors influence simple arithmetic performance at early stages of learning, whereas the performance of highly practiced adults is influenced by problem size (see, e.g., Geary, 1996), we believe that the influence of experiential factors never disappears completely. Practice can influence both strategy selection and strategy efficiency in children (see, e.g., Siegler, 1986) and young adults (the present study). We therefore believe that future research should not confine itself to the investigation of skill variables but should also concentrate on other individual differences, such as daily arithmetic practice. More generally, and in agreement with other researchers (e.g., Kirk \& Ashcraft, 2001; LeFevre, Sadesky, \& Bisanz, 1996), we believe that investigations of individual differences and their relationship to online performance should be a priority for the field.

Note-This article was accepted by the previous editorial team, when Colin M. MacLeod was Editor.

\section{AUTHOR NOTE}

The research reported in this article was supported by Grant 011D07803 of the Special Research Fund at Ghent University to the first author and by Grant 10251101 of the Special Research Fund at Ghent University to the second author. Thanks are extended to the Immaculata Instituut in De Panne, Belgium, where all experiments were administered, and to David Geary and two anonymous reviewers for their helpful comments on previous drafts of this article. Correspondence concerning this article should be addressed to I. Imbo, Department of Experimental Psychology, Ghent University, Henri Dunantlaan 2, B-9000 Ghent, Belgium (e-mail: ineke.imbo@ugent.be).

\section{REFERENCES}

AshCRAFT, M. H. (1987). Children's knowledge of simple arithmetic: A developmental model and simulation. In J. Bisanz, C. J. Brainerd, \& R. Kail (Eds.), Formal methods in developmental psychology: Progress in cognitive development research (pp. 302-338). New York: Springer.

AshCRAFT, M. H. (1992). Cognitive arithmetic: A review of data and theory. Cognition, 44, 75-106.

AshCRAFT, M. H. (1995). Cognitive psychology and simple arithmetic: A review and summary of new directions. Mathematical Cognition, 1, 3-34.

Ashcraft, M. H., Donley, R. D., Halas, M. A., \& Vakali, M. (1992). Working memory, automaticity, and problem difficulty. In J. I. D. Campbell (Ed.), The nature and origins of mathematical skills (pp. 301-329). Amsterdam: North-Holland. 
Ashcraft, M. H., \& Faust, M. W. (1994). Mathematics anxiety and mental arithmetic performance: An exploratory investigation. Cognition \& Emotion, 8, 97-125.

Ashcraft, M. H., \& KIRK, E. P. (2001). The relationships among working memory, math anxiety, and performance. Journal of Experimental Psychology: General, 130, 224-237.

Baroody, A. J. (1994). An evaluation of evidence supporting factretrieval models. Learning \& Individual Differences, 6, 1-36.

CAmpbell, J. I. D. (1987a). Network interference and mental multiplication. Journal of Experimental Psychology: Learning, Memory, \& Cognition, 13, 109-123.

Campbell, J. I. D. (1987b). Production, verification, and priming of multiplication facts. Memory \& Cognition, 15, 349-364.

CAmpbell, J. I. D. (1995). Mechanisms of simple addition and multiplication: A modified network-interference theory and simulation. Mathematical Cognition, 1, 121-164.

Campbell, J. I. D., \& Austin, S. (2002). Effects of response time deadlines on adults' strategy choices for simple addition. Memory \& Cognition, 30, 988-994.

Campbell, J. I. D., \& Graham, D. J. (1985). Mental multiplication skill: Structure, process, and acquisition. Canadian Journal of Psychology, 39, 338-366.

Campbell, J. I. D., \& Gunter, R. (2002). Calculation, culture, and the repeated operand effect. Cognition, 86, 71-96.

Campbell, J. I. D., \& Oliphant, M. (1992). Representation and retrieval of arithmetic facts: A network-interference model and simulation. In J. I. D. Campbell (Ed.), The nature and origins of mathematical skills (pp. 331-364). Amsterdam: North-Holland.

Campbell, J. I. D., \& Timm, J. C. (2000). Adults' strategy choices for simple addition: Effects of retrieval interference. Psychonomic Bulletin \& Review, 7, 692-699.

Campbell, J. I. D., \& Xue, Q. (2001). Cognitive arithmetic across cultures. Journal of Experimental Psychology: General, 130, 299-315.

CARr, M., \& DAVIS, H. (2001). Gender differences in arithmetic strategy use: A function of skill and preference. Contemporary Educational Psychology, 26, 330-347.

CARR, M., \& Jessup, D. L. (1997). Gender differences in first-grade mathematics strategy use: Social and metacognitive influences. Journal of Educational Psychology, 89, 318-328.

CARr, M., Jessup, D. L., \& Fuller, D. (1999). Gender differences in first-grade mathematics strategy use: Parent and teacher contributions. Journal for Research in Mathematics Education, 30, 20-46.

Chen, C., \& UtTal, D. H. (1988). Cultural values, parents' beliefs, and children's achievement in the United States and China. Human Development, 31, 351-358.

Ekstrom, R. B., French, J. W., \& Harman, H. H. (1976). Manual for kit of factor-referenced cognitive tests: 1976. Princeton, NJ: Educational Testing Service.

Faust, M. W., AshCraft, M. H., \& Fleck, D. E. (1996). Mathematics anxiety effects in simple and complex addition. Mathematical Cognition, 2, 25-62.

Fennema, E., Carpenter, T. P., Jacobs, V. R., Franke, M. L., \& Levi, L. W. (1998). A longitudinal study of gender differences in young children's mathematical thinking. Educational Researcher, 27, 6-11.

French, J. W., Ekstrom, R. B., \& Price, I. A. (1963). Kit of reference tests for cognitive factors. Princeton, NJ: Educational Testing Service.

GEARY, D. C. (1994). Children's mathematical development: Research and practical applications. Washington, DC: American Psychological Association.

GEARY, D. C. (1996). The problem-size effect in mental addition: Developmental and cross-national trends. Mathematical Cognition, 2, 63-93.

Geary, D. C. (1999). Sex differences in mathematical abilities: Commentary on the math-fact retrieval hypothesis. Contemporary Educational Psychology, 24, 267-274.

Geary, D. C., Bow-Thomas, C. C., Liu, F., \& Siegler, R. S. (1996). Development of arithmetical competencies in Chinese and American children: Influence of age, language, and schooling. Child Development, 67, 2022-2044.

Geary, D. C., Frensch, P. A., \& Wiley, J. G. (1993). Simple and complex mental subtraction: Strategy choice and speed-of-processing differences in younger and older adults. Journal of Experimental Child Psychology, 47, 175-192.
Geary, D. C., Hamson, C. O., Chen, G.-P., Liu, F., Hoard, M. K., \& Salthouse, T. A. (1997). Computational and reasoning abilities in arithmetic: Cross-generational change in China and the United States. Psychonomic Bulletin \& Review, 4, 425-430.

Geary, D. C., Liu, F., \& Bow-Thomas, C. C. (1992). Numerical cognition: Loci of ability differences comparing children from China and the United States. Psychological Science, 3, 180-185.

Geary, D. C., Salthouse, T. A., Chen, G.-P., \& Liu, F. (1996). Are East Asian versus American differences in arithmetic ability a recent phenomenon? Developmental Psychology, 32, 254-262.

Geary, D. C., Saults, S. J., LiU, F., \& Hoard, M. K. (2000). Sex differences in spatial cognition, computational fluency, and arithmetical reasoning. Journal of Experimental Child Psychology, 77, 337-353.

Geary, D. C., \& Widaman, K. F. (1987). Individual differences in cognitive arithmetic. Journal of Experimental Psychology: General, 116, 154-171.

Geary, D. C., \& Widaman, K. F. (1992). Numerical cognition: Convergence of componential and psychometric models. Intelligence, 16, 47-80.

Geary, D. C., \& Wiley, J. G. (1991). Cognitive addition: Strategy choice and speed of processing differences in young and elderly adults. Psychology \& Aging, 6, 474-483.

Gilles, P.-Y., Masse, C., \& Lemaire, P. (2001). Différences individuelles dans l'utilisation de stratégies en arithmétique. L'Année Psychologique, 101, 9-32.

Неснт, S. A. (1999). Individual solution processes while solving addition and multiplication math facts in adults. Memory \& Cognition, 27, 1097-1107.

KAYE, D. B. (1986). The development of mathematical cognition. Cognitive Development, 1, 157-170.

Kaye, D. B., DeWinstanley, P., Chen, Q., \& Bonnefil, V. (1989). Development of efficient arithmetic computation. Journal of Educational Psychology, 81, 467-480.

KIRK, E. P., \& AshCraft, M. H. (2001). Telling stories: The perils and promise of using verbal reports to study math strategies. Journal of Experimental Psychology: Learning, Memory, \& Cognition, 27, 157-175.

Lebiere, C., \& Anderson, J. R. (1998). Cognitive arithmetic. In J. R. Anderson \& C. Lebiere (Eds.), The atomic components of thought (pp. 297-342). Mahwah, NJ: Erlbaum.

LeFeVre, J.-A., \& BisAnZ, J. (1986). A cognitive analysis of numberseries problems: Sources of individual differences in performance. Memory \& Cognition, 14, 287-298.

LeFevre, J.-A., Bisanz, J., Daley, K. E., Buffone, L., Greenham, S. L., \& SADESKY, G. S. (1996). Multiple routes to solution of singledigit multiplication problems. Journal of Experimental Psychology: General, 125, 284-306.

LeFevre, J.-A., \& KulaK, A. G. (1994). Individual differences in the obligatory activation of addition facts. Memory \& Cognition, 22, 188-200.

LeFevre, J.-A., KulaK, A. G., \& Bisanz, J. (1991). Individual differences and developmental change in the associative relations among numbers. Journal of Experimental Child Psychology, 52, 256-274.

LeFeVre, J.-A., \& LIU, J. (1997). The role of experience in numerical skill: Multiplication performance in adults from Canada and China. Mathematical Cognition, 3, 31-62.

LeFevre, J.-A., Sadesky, G. S., \& Bisanz, J. (1996). Selection of procedures in mental addition: Reassessing the problem size effect in adults. Journal of Experimental Psychology: Learning, Memory, \& Cognition, 22, 216-230.

McCloskey, M. (1992). Cognitive mechanisms in numerical processing: Evidence from acquired dyscalculia. Cognition, 44, 107-157.

Penner-Wilger, M., Leth-Steensen, C., \& LeFevre, J.-A. (2002). Decomposing the problem-size effect: A comparison of response time distributions across cultures. Memory \& Cognition, 30, 1160-1167.

Roussel, J.-L., Fayol, M., \& Barrouillet, P. (2002). Procedural vs. direct retrieval strategies in arithmetic: A comparison between additive and multiplicative problem solving. European Journal of Cognitive Psychology, 14, 61-104.

Royer, J. M., Tronsky, L. N., Chan, Y., Jackson, S. J., \& Marchant, H., III (1999). Math-fact retrieval and the cognitive mechanism underlying gender differences in math test performance. Contemporary Educational Psychology, 24, 181-266.

Seyler, D. J., Kirk, E. P., \& Ashcraft, M. H. (2003). Elementary sub- 
traction. Journal of Experimental Psychology: Learning, Memory, \& Cognition, 29, 1339-1352.

Siegler, R. S. (1986). Unities across domains in children's strategy choices. In M. Perlmutter (Ed.), Minnesota Symposia on Child Psychology: Vol. 19. Perspectives on intellectual development (pp. 1-48). Hillsdale, NJ: Erlbaum.

SiEgLER, R. S. (1988). Strategy choice procedures and the development of multiplication skill. Journal of Experimental Psychology: General, 117, 258-275.

SIEGLER, R. S. (1989). Hazards of mental chronometry: An example from children's subtraction. Journal of Educational Psychology, 81, 497-506.

Siegler, R. S., \& Shipley, E. (1995). Variation, selection, and cognitive change. In G. Halford \& T. Simon (Eds.), Developing cognitive competence: New approaches to process modeling (pp. 31-76). Hillsdale, NJ: Erlbaum.

Stevenson, H. W., Chen, C., \& Lee, S.-Y. (1993). Mathematics achievement of Chinese, Japanese, and American children: Ten years later. Science, 259, 53-58.

Stevenson, H. W., Lee, S.-Y., Chen, C., Lummis, M., Stigler, J., Liu, F., \& Ge, F. (1990). Mathematics achievement of children in China and the United States. Child Development, 61, 1053-1066.

Stevenson, H. W., Lee, S.-Y., \& Stigler, J. W. (1986). Mathematics achievement of Chinese, Japanese, and American children. Science, 231, 693-699.

Thimbleby, H. (2000). Calculators are needlessly bad. International Journal of Human-Computer Studies, 52, 1031-1069.

Widaman, K. F., \& Little, T. D. (1992). The development of skill in mental arithmetic: An individual differences perspective. In J. I. D. Campbell (Ed.), The nature and origins of mathematical skills (pp. 189-253). Amsterdam: North-Holland.

ZBrodoff, N. J. (1995). Why is $9+7$ harder than $2+3$ ? Strength and interference as explanations of the problem-size effect. Memory \& Cognition, 23, 689-700.

\section{NOTES}

1. In the present article, the term individual differences is used to refer to differences inherent in individuals (e.g., gender), as well as to differences resulting from the environment (e.g., arithmetic practice, calculator use).

2. In Belgium, high school education starts at 12 years of age and normally takes 6 years to complete. After their 2 nd year of high school, students have to choose among different study options, such as humanities, economics, languages, mathematics, or sciences. The amount of students' daily arithmetic practice (defined as the number of hours per week dedicated to math and science classes) varies across these study options. Specifically, students enrolled in a mathematics curriculum have more arithmetic-related classes than students in an economics curriculum, who in turn have more arithmetic-related classes than students in a humanities curriculum.

3. It should be noted that some predictor variables correlated with each other. Specifically, the correlations between daily arithmetic practice and arithmetic skill, between calculator use and arithmetic skill, and between daily arithmetic practice and calculator use were significant ( $r=.256, r=-.347$, and $r=-.297$, respectively). These correlations are not problematic for the regression analyses, however. Indeed, omitting one of these predictors in the regression model did not result in dramatic changes of the parameter estimates or of the significance results for the remaining predictors.

(Manuscript received October 15, 2004; revision accepted for publication February 4, 2006.) 